\title{
A Description-Based Mashup for Cooperation of Mobile Devices
}

\author{
Korawit Prutsachainimmit and Takehiro Tokuda \\ Department of Computer Science, Tokyo Institute of Technology \\ Meguro, Tokyo 152-8552, Japan \\ \{korawit, tokuda\}att.cs.titech.ac.jp
}

\begin{abstract}
Recently, the study of mobile mashup has become important in mobile computing. Mashup approaches were proposed to allow users to create mashup for mobile devices. However, existing approaches still lack attention to enable mashup for cooperation of mobile devices. We present a descriptionbased mashup construction approach for cooperation of mobile devices. Our approach allows end-users to create mobile mashup applications by integrating mobile applications, Web applications and Web services. We define a mashup description language for describing logic of mashup. We use a mashup generator to simplify mashup construction. We also present a mashup execution environment to automate information sharing for cooperation of mobile devices.
\end{abstract}

Keywords: Mobile mashup, Cooperation mashup, Description language.

\section{Introduction}

Mobile mashup has recently become an important trend in mobile computing. Mashup solutions and tools emerged to assist end-users to compose mashup applications for mobile environment. Yahoo! Pipes and Intel Mash Maker focus on data integration and filtering. TELAR mashup platform [1] presents a way to combine mobile devices' features such as GPS with existing Web resources. Kaltofen et al. presents an end-users' mobile mashup for cross-platform deployment [2]. Most of the proposed solutions share a common characteristic where they focus on mashup development for single device. The proposed solutions also have limited capabilities to develop mashup for cooperation of multiple devices.

The usage style of mobile application is changing from individual use to collaborative use. Collaborative applications such as groupware or social applications which are commonly used on desktop computers are adapted to the mobile platform. Hence, mashup development for cooperation of mobile devices is taken into account. With cooperation of devices, different information from multiple mobile devices can be integrated with existing mashup components to produce new mashup output. Thus, mashup with cooperation of mobile devices is an interesting research topic in mobile mashup. 
In our previous work [3], we proposed a mobile mashup approach for end-users by using a description language and a mashup generator. We also presented Tethered Web service (TeWS) to support cooperation of mobile devices. However, the previous work still has limitations. Then, this research aims to find an optimal mashup approach for cooperation of mobile devices. To achieve this goal, the following objectives were set:

1. To develop a mobile mashup construction system dealing with cooperation of mobile devices.

2. To enable integration of mobile applications, Web applications and Web services in flow-based and event-based execution.

3. To allow automatic generation of typical database mobile applications for mashup.

4. To analyze and optimize efficiency of the mashup approach.

5. To evaluate usability of the mashup approach by conducting user-based test.

\section{Mashup Approach}

The general concept of our approach is using a description language for mashup construction. The description language allows mashup composers to define mashup components and detail of its integration. The mashup components can be Web applications, Web services and mobile applications. We also apply the descriptionbased construction technique to create typical data-centric mobile applications which can be integrated into mashup. To leverage mashup composition effort, we use a GUI mashup designer tool to help mashup composers in creating mashup description files. The description files will be used with a mashup generator to build mashup applications. To enable cooperation of devices, we use a mashup execution environment which helps in data exchanging among participating devices.

\subsection{Mashup Description Language}

The mashup description language is an XML-based description language which is designed for describing mashup components and its integration. The description language provides a way to describe mashup components which can be used in a mashup application. Mashup components consist of:

1. Web Application Component (WA). This component can extract a part of a Web page or a query through an HTML form. Mashup composers are provided with a Web extraction assistant tool [4] to indicate part of required information on a Web page. The description of this component will be generated to JavaScript code and executed in the runtime environment on a mobile device.

2. Web Service Component (WS). This component is used for describing a Web service API. The target Web service will be invoked to extract a whole or a part of the result. 
3. Mobile Application Component (MA). This component allows a mobile application which implemented Intent and Service [5] messaging protocol to be integrated in mashup.

4. Mobile Application Generator (MG). To expand integration capability of mobile applications, this component helps composers in creating a typical data-centric mobile application by using a description. The generated applications can be integrated and reused as a mobile application.

5. Data Manipulation Component (DM). Output from one or more components can be transformed and filtered by using this component.

6. Cooperation Component (CC). This component will be used for cooperation of multiple devices. Required information from participating devices can be described in this component. The description of this component will be generated to code for communicating with the execution environment to exchange information with other devices.

\subsection{Mashup Construction Process}

The mashup construction process is shown in Figure 1. To compose a mashup application, a mashup composer creates an abstract model of mashup by defining components and detail of its integration. The mashup designer tool will assist the mashup composer in transforming the abstract model into the description language. Output from the mashup designer tool is an XML description file. The description file will be used as an input of the mashup generator to generate Java source code. This generated code will be compiled into a mobile application which can be deployed on target devices. The mashup application can be used as an ordinary mobile application.

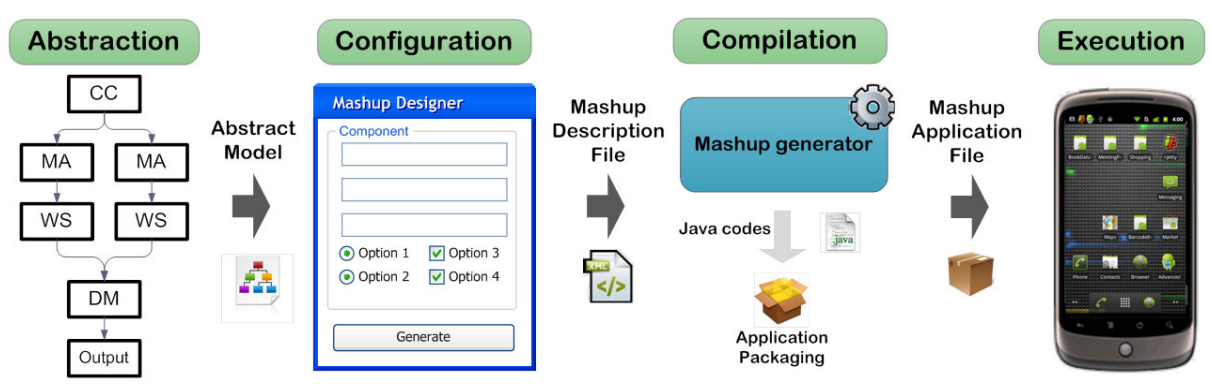

Fig. 1. Mashup Construction Process

\subsection{Mashup Execution Environment}

To achieve mashup for cooperation of devices, participating devices need a capability to communicate with other devices for exchanging mashup required information. We use an execution environment to automate this task. Our execution environment allows exchanging information between devices by using a custom mobile application 
called Cooperation Agent. The cooperation agent will be installed on the participating devices to take care of devices' connectivity and information sharing. With the cooperation agent, the participating devices can send messages to request mashup required information from other devices. To reduce data transmission among devices, messages that are sent in our execution environment use RESTful Web services and JSON [6].

\section{Mashup for Cooperation of Mobile Devices}

To demonstrate a mashup for cooperation of mobile devices, we implemented a mashup scenario with our approach. This scenario simulates a shopping situation in a department store for 3 or more users. Mashup for this scenario will help users in comparing prices on a local store with prices from online stores. The mashup also creates a summary of selected products shared to all users.

We show mashup model and screenshots in Figure 2. For this mashup, the cooperation component requests for a barcode from all client devices. Each client device reads a barcode of a selected product and submits it to the server device. Each barcode is given to the Web service component of Google's Search API for Shopping [7] to find available online stores and prices. The data manipulation component extracts the lowest price and stores' information. The lowest price is converted to target currency with Web services component of Exchange Rate API [8]. The converted price and stores' information are combined into a list on the server. After all clients finish sending the barcodes, the summary list of selected products can be shared among all devices.



Fig. 2. Mashup model and screenshots of Shopping Assistance 
In order to study cooperation of different mobile platforms, this example is implemented by using an Android device as the server and iOS devices as the clients. The mashup application was generated and deployed in the server device while the cooperation agent is pre-installed to all clients device. Mashup users can use this mashup application as an ordinary mobile application.

\section{Conclusion and Future Work}

This paper has presented goals and the current state of our research. We propose a description-based solution for mashup construction on single and cooperation of devices. The mashup created by our approach is capable for integration of mobile applications and Web information. We have also presented a solution for devices' connectivity by using the mashup execution environment. We have demonstrated capabilities of our approach with the example scenario. However, our current work is designed for flow-based mashup, where the mashup components are executed in sequences. In addition, some parts of mashup construction still require manual operations.

Our future research is targeted towards designing, implementing, and evaluating a novel mashup construction system for cooperation of mobile devices. The following specific contributions are planned:

- To enable event-based mashup where mashup components are executed by events.

- To allow automatic generation of typical database mobile applications in mashup.

- To analyze usability by conducting a user-based evaluation.

- To optimize efficiency of mashup execution on mobile devices.

\section{References}

1. Brodt, A., Nicklas, D., Sathish, S., Mitschang, B.: Context-Aware Mashups for Mobile Devices. In: Bailey, J., Maier, D., Schewe, K.-D., Thalheim, B., Wang, X.S. (eds.) WISE 2008. LNCS, vol. 5175, pp. 280-291. Springer, Heidelberg (2008)

2. Kaltofen, S., Milrad, M., Kurti, A.: A Cross-Platform Software System to Create and Deploy Mobile Mashups. In: Benatallah, B., Casati, F., Kappel, G., Rossi, G. (eds.) ICWE 2010. LNCS, vol. 6189, pp. 518-521. Springer, Heidelberg (2010)

3. Chaisatien, P., Prutsachainimmit, K., Tokuda, T.: Mobile Mashup Generator System for Cooperative Applications of Different Mobile Devices. In: Auer, S., Díaz, O., Papadopoulos, G.A. (eds.) ICWE 2011. LNCS, vol. 6757, pp. 182-197. Springer, Heidelberg (2011)

4. Guo, J., Chaisatien, P., Han, H., Noro, T., Tokuda, T.: Partial Information Extraction Approach to Lightweight Integration on the Web. In: Daniel, F., Facca, F.M. (eds.) ICWE 2010. LNCS, vol. 6385, pp. 372-383. Springer, Heidelberg (2010)

5. Android Developers, http://developer.android.com/index.html

6. Tsai, C.-L., Chen, H.-W., Huang, J.-L., Hu, C.-L.: Transmission reduction between mobile phone applications and RESTful APIs. In: Proceedings of the 2011 ACM Symposium on Applied Computing, SAC 2011 (2011)

7. Search API for Shopping, http://code.google.com/apis/shopping/search/

8. Exchange Rate API, http: / /www . exchangerate-api.com/ 